\title{
Design and implementation of heterogeneous surface gateway for underwater acoustic sensor network
}

\author{
Youngjun $\mathrm{Jo}^{1}$, Sangsoon $\mathrm{Lim}^{2}$ \\ ${ }^{1}$ LG Electronics, Seoul, South Korea \\ ${ }^{2}$ Department of Computer Science Engineering, Sungkyul University, Anyang, South Korea
}

\begin{abstract}
Article Info
Article history:

Received Apr 24, 2018

Revised Sep 27, 2018

Accepted Oct 15, 2018

Keywords:

Architecture

Sensor networks

Surface gateway

ABSTRACT

Underwater Acoustic Sensor Networks (UASNs) are used for diverse purposes such as pollution monitoring, disaster prevention and industrial sensing in the oceans. Especially, UASNs are mainly focusing on monitoring various underwater environmental data and delivering the data to a monitoring center where nearby or far from the deployed area. To reliably deliver the data, a surface gateway should convert acoustic signal to RF (Radio Frequency) signal. In this paper, we devise a multiple interfacesbased surface gateway that can connect both a cellular network and a Zigbee network. Depends on the service requirement, the surface gateway can easily adopt each wireless interface and relay the data to a low power ZigBee network or a long range CDMA network.
\end{abstract}

Underwater
Copyright $(0) 2019$ Institute of Advanced Engineering and Science. All rights reserved.

\section{Corresponding Author:}

Sangsoon Lim,

Department of Computer Science Engineering,

Sungkyul University,

Anyang, South Korea.

Email:1ssgood80@gmail.com

\section{INTRODUCTION}

Recent interest in water resources has led to active research on Underwater Acoustic Sensor Networks (UASNs). About $70 \%$ of the surface of the earth is covered with water and various resources are still untouched. The UASNs are deployed in such an unexplored area to provide important functions such as pollution monitoring, marine condition monitoring, emergency disaster prevention and so on. The following are the main applications of UASNs [1], [2].

UASNs can sense polluted environments and track fishes and micro-organisms with small sensor nodes. Each sensor node periodically measures various information and sends them to the monitoring center for the purpose of an environmental monitoring. In addition, UASNs can alert a critical problem in the ocean for a disaster preventions. They gather crustal movement information from several remote locations. Based on the information, they can provide tsunami warnings to the shore areas or analyze the effects of seaquakes. In the case of assisted navigation, each sensor node can recognize various dangerous scenarios on the seabed. It detects rocks and shoals in shallow waters. UASNs can be connected to a remote robot when the robot explores the sea. In this case, each sensor node can transmit various control information to the robots and help them to complete their tasks.

Especially, underwater sensor nodes are widely deployed in the ocean field to cover a large part of the area. It means that many sensor nodes are located far from a seashore, and thus a surface gateway needs to convey various monitoring information with a long range wireless transmission technology. To satisfy service requirements, we devise a multiple interfaces-based surface gateway that can connect both a cellular network and a Zigbee network. Each underwater sensor node senses the physical information and transmit them to its neighbor sensor nodes with a low power ZigBee network. In addition, neighbor nodes aggregate 
those information and relay them to a specific surface gateway. The surface gateway gather all information from other sensor nodes and reliably transfer those information to the monitoring center. In this case, Code Division Multiple Access (CDMA) is highly recommended for an additional wireless interface due to its robustness in the presence of signal fading[1]. Moreover, the communication range of CDMA is much larger than other wireless technologies such as Wi-Fi [3], Bluetooth, and ZigBee [4]. In the case of ZigBee, usally used in Wireless Sensor Networks (WSNs) [5-6], the transmission range is not enough to convey the ocean information to the monitoring center. Otherwise, the transmission range of CDMA is up to $1 / 2$ mile in cities, and it can be extended up to 5 miles in rural areas. By adopting the additional CDMA interface to a surface gateway, UASNs can communicate with a low power ZigBee among other sensor nodes and the gateway directly connect to the monitoring center without unnecassry relay protocols.

The remainder of this paper is organized as follows. Section 2 briefly introduce the related work. We then describe the overall architecture of surface gateway in Section 3. Finally, Section 4 concludes the paper.

\section{RELATED WORK}

In this section, we introduce the overall architecture of UASNs. Especially, we describe the basic concept of UASNs with their various components and detailed operations. The UASNs consists of many tiny underwater sensor nodes, underwater sink nodes and surface gateways. Each tiny sensor node performs an environmental monitoring with several underwater sensor modules that collect their surrounding environment information. In order to communicate with neighboring nodes under the water, it conveys measured information to the sink node via underwater acoustic modem that converts a basic digital signal to an ultrasonic signal. After receiving raw datas, the underwater sink node checks the ocean data and selectively sends them to the surface gateway. After that, the surface gateway takes a role of a general relay node between UASNs and infrastructure such as an access point or a base station [2].

In order to support large coverage of UASNs, the communication range of surface gateway is one of the major concerns. In case of WSNs, they employ a low power communication technology, called ZigBee to reduce the energy consumption of sensor nodes. It means that a traditional sensor network has the limitation when they share monitoring information among other clusters of sensor networks. In case of UASNs, due to their deployment policies, the surface gateway is often far from the base station. Thus, they have to support both a low power communication among other clusters and a long range communication for the base station. However, various previous approaches mainly consider the low power communication. To remedy the problem, our surface gateway exchanges its aggregated information via a CDMA interface.

\section{DESIGN OF HETEROGENEOUS SURFACE GATEWAY}

\subsection{The overall components of surface gateway}

The main components of the surface gateway are a microcontroller module, a CDMA communication module and a RF module. Figure 1 illustrates the block diagram of the surface gateway.

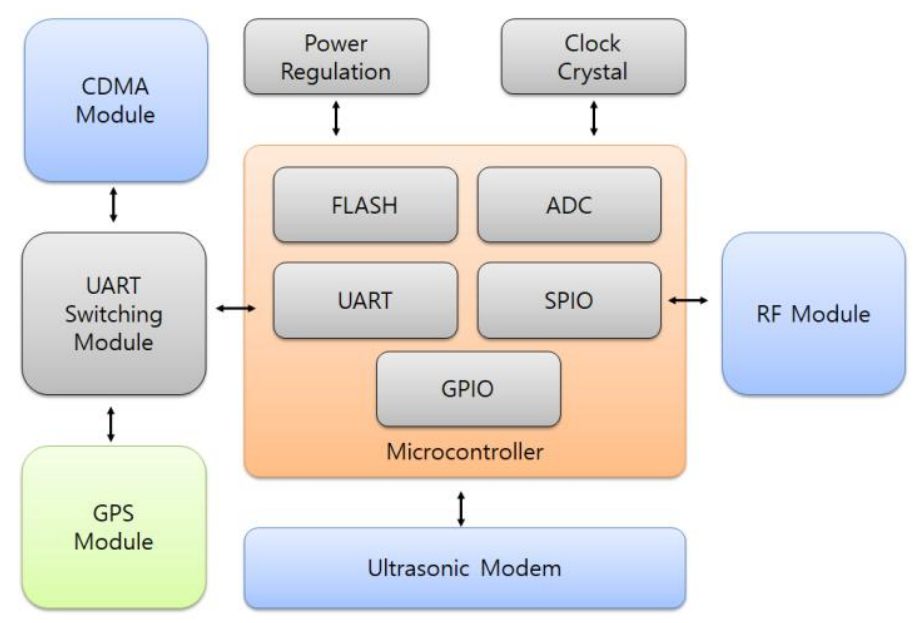

Figure 1. Block diagram of surface gateway 
The gateway is equipped with the Atmega128L as a Microcontroller Unit (MCU). The Atmega128L is the one of the popular low power 8-bit microcontrollers to control the entire system. It consists of $128 \mathrm{kbytes}$ flash, dual programmable UARTs and a SPI interface. One channel of the UART is connected to the serial port on the computer to debug the device. The other one is connected to the CDMA module and the GPS module via UART swithcing module to avoid severe collisions between both modules.

We use the Qualcomm MSM6050 chipset as a CDMA module. It can support CDMA 2000 protocol and various interfaces such as UART ports, GPIO and an antenna. As shown in the Figure 2, the CDMA connectivity can transfer the processed datas from an access area to the cellular network with a long range communication. Based on the CDMA protocol, the CDMA modules establishes the basic connection between the surface gateway and the monitoring center. After establishing the connection, each module transmits the datas with the regular CDMA protocol via a duplex link. To disconnect the communication link, one module sends a disconnection command. After receiving the command, the destination module transmits a response message to completely quit the connection. In addition, when the timer is expierd, they are disconnected. The CDMA module starts a connection timer after successfully connecting each other. Once the timer is expired, one module automatically sends a 'No carrier' message to the destination module to disconnect the link.

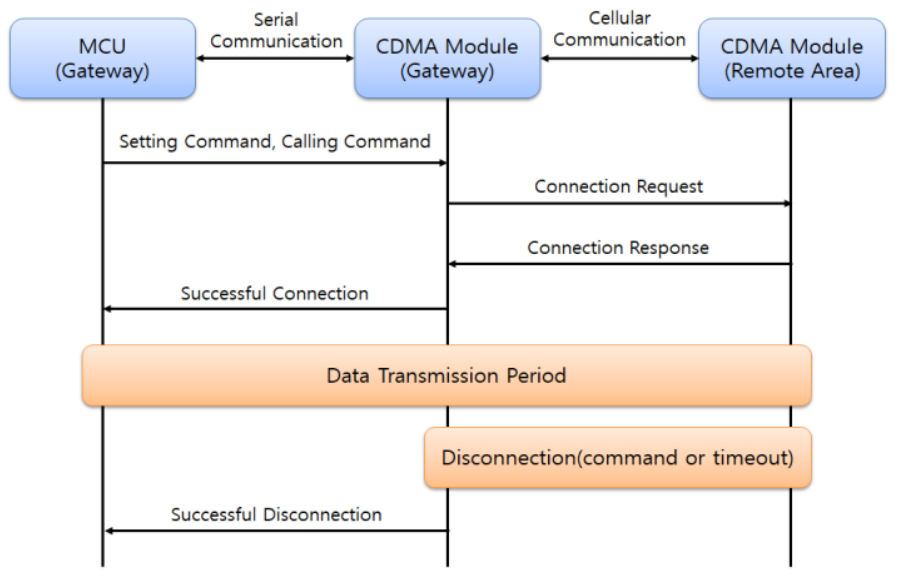

Figure 2. CDMA communication flows

As shown in the Figure 3 we implement the prototype of the surface gateway. The surface gatewway consists of four areas. The first area is MCU and second area is CDMA module. The third area is the GPS module and the fourth area is the RF module. We use CC2420 RFIC as the RF module to support the ZigBee protocol. It provides an energy efficiency communication among the surface gateways and tiny sensor nodes. If the distance between the surface gateway and the monitoring center is within the transmission range of the ZigBee connectivity, it can also support the data exchange between them. In addition, we set a GPS module for the purpose of the location based service. We use MTK MT3318 chipset to support multi-channel and high accuracy positioning service. The GPS module periodically transmits location informatin with longitude, latitude, time, a number of satellites and so on. We obtain latitude and longitude from the location information and calculate the exact position of the node.

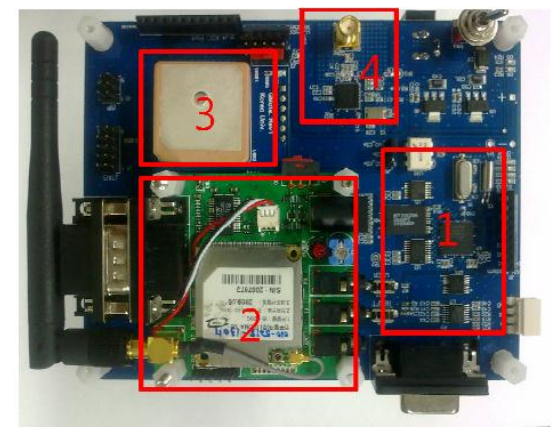

Figure 3. The prototype of the surface gateway 


\subsection{The basic operation flow of surface gateway}

In order to control the underwater robot through the acoustic modem and the CDMA module, we design the basic operation flow of surface gateway. The detailed operation of the surface gateway depends on the MAC protocol. In our experimentation, we mainly focus on the TDMA based MAC protocol. It periodically conveys wakeup schedules via a beacon message. Based on the message, each node can set the transmission timing for underwater robot control messages and data exchanges.

Figure 4 shows the basic operation flow of the acoustic modem. If the acoustic modem receives the messages, it checks the presence of the beacon message. If it contains the beacon message, the gateway extracts a time schedule. Based on the time schedule, it determines whether sending control messages to the sink node or not. To reliably control the robot, the gateway periodically sends robot control message with its buffer information. In addition, in the data gathering phase, the surface gateway listens for the message from the acoustic modem. After receiving some gathered data from the underwater sink node, the surface gateway sends it to the monitoring center through CDMA module. In this case, we can dynamically adjust the control period and information sharing period. If we detects a specific event in the ocean spot, we increase the information sharing period to gether more information and convey them to the monitoring center.

Figure 5 shows the basic operation flow of CDMA module. In the initial phase, the gateway controls its CDMA module to connect to the remote monitoring center. After that, it waits for the incoming robot control messages from the CDMA module. When the control message arrives from the monitoring center, the MCU keeps the messages for a while. While saving the robot control messages, it tries to send them to the underwater sink node. If the sending operation fails, the node clears the buffer containing control messages. If the control message is dropped in the surface gateway, it notifies the status of the control message to the monitoring center. Once the monitoring center detects the loss of control messages, it successively retransmits the original control message to the surface gateway to support a reliable transmission. However, excessive retransmission can degrade the overall performance of the system. Thus, the monitoring center adaptively change the retransmission count according to the wireless channel status. When they experience severe interference from diverse environmental factors, the center decreases the retransmission trials to reduce unnecessary transmissions.

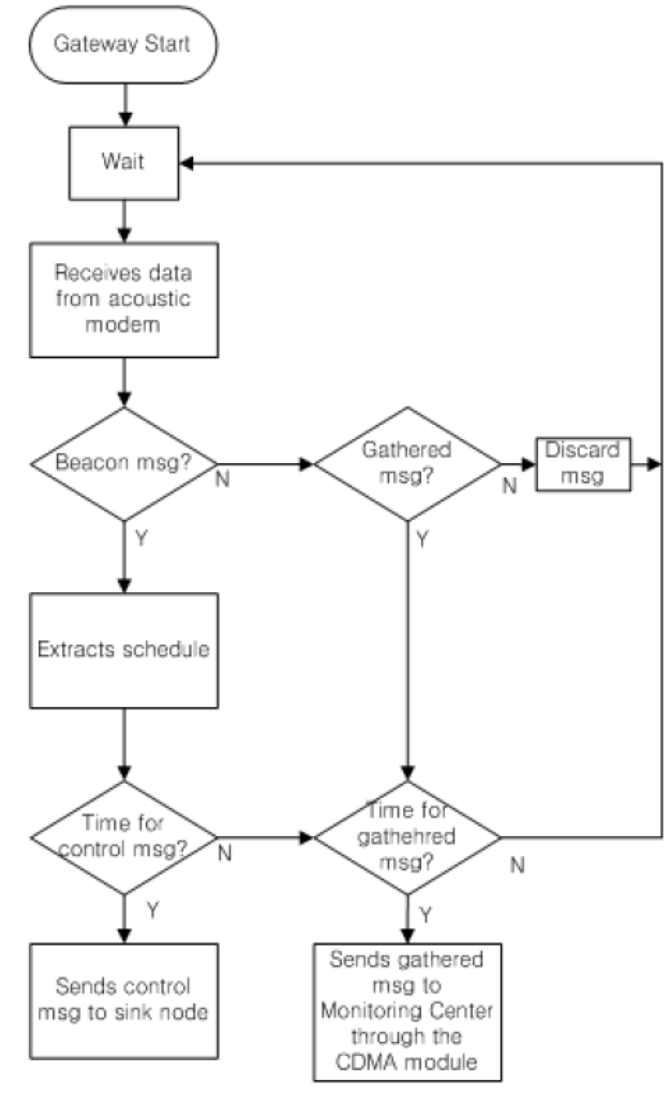

Figure 4. The operation flow of the acoustic modem

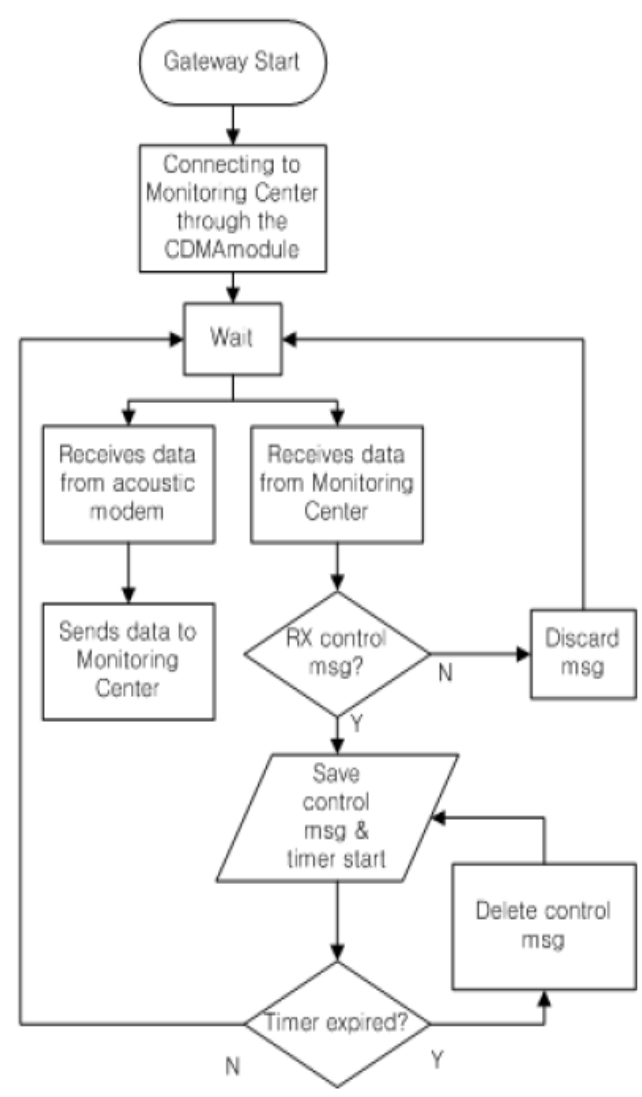

Figure 5. The operation flow of the CDMA module 


\subsection{Low power information sharing of surface gateway}

The surface gateway can use a low power ZigBee interface to communicate other sensor clusters. Due to the low power requirement, the gateway selectively use both the CDMA module and the ZigBee module. The energy consumption of the CDMA module is much higher than the ZigBee module. For this reason, the gateway shares basic control informaion among sensor clusters via the ZigBee module. When we perform low power information sharing, we should consider the cross-technology interference (CTI) [7]-[9] from other wireless technologies such as Wi-Fi, Bluetooth and Bluetooth Low Energy (BLE). If other devices close to the gateway exchange their informaion through those wireless technologies, they aggrevate the performance of the low power communication. To investigate the interference, we measure Packet Delivery Ratio (PDR) of a ZigBee interface in our lab environment. As shown in the Figure 6 we use a traditional wireless sensor node, called telosB [10], [11] to measure a normal ZigBee performance. Figure 7 shows the interference result of Wi-Fi clients. As the number of Wi-Fi clients increases, the PDR of ZigBee interfaces are decreased due to the cross-technology interference. To avoid the interference problem in the surface gateway, we periodically change the operation channel of ZigBee interface after measuring the interface level.

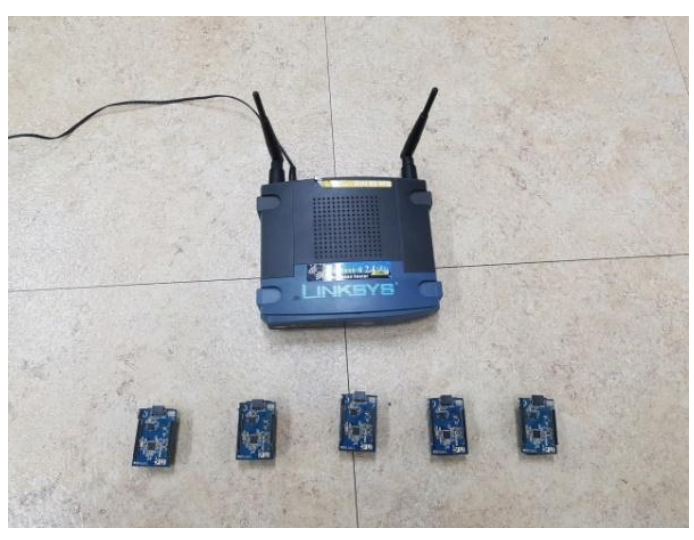

Figure 6. Experimental setup-interference measurement

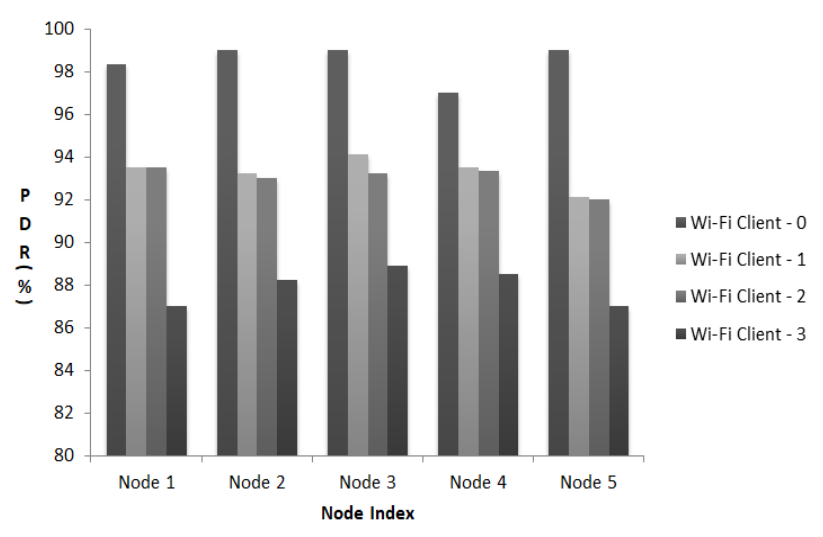

Figure 7. Packer delivery ratio of a ZigBee interface

\section{CONCLUSION}

In this paper, we introduced the heterogeneous architecture of the surface gateway. The architecture has multiple wireless interfaces to provide reliable and efficient connectivity. In addition, we proposed the surface gateway operation flows with multiple interfaces. Our prototype can support a long range communication with the cellular network providing wider and stronger connection which is possible for reliable connectivity between the surface gateway and monitoring center. In addition, our prototype can also support the low power information sharing among surface gateway and tiny sensor nodes. This paper mainly focus on the cellular and ZigBee network. However, if we embed another module such as WLAN, we can cover various UASNs scenarios. In addition, considering mobility, an automatically searching the appropriate wireless channel mechanism is needed for mobile gateway for efficient data transmission in the future.

\section{ACKNOWLEDGEMENTS}

This work was supported by the National Research Foundation of Korea(NRF) grant funded by the Korea government(MSIT) (No. NRF-2018R1C1B5038818).

\section{REFERENCES}

[1] I. F. Akyildiz, D. Pompili, and T. Melodia, "Underwater Acoustic Sensor Networks: Research Challenges," Ad Hoc Networks, pp.257-279, May 2005.

[2] Jim Partan et al., "A Survey of Practical Issues in Underwater Networks", In proc. ACM International Workshop on Underwater Networks (WUWNet)

[3] “Wireless LAN Medium Access Control (MAC) and Physical: Specications," IEEE Std 802.11, 2007.

[4] "Wireless Medium Access Control (MAC) and Physical Layer (PHY) Specications for Low-Rate Wireless Personal Area Networks (LR-WPANs)," IEEE Std. 802.15.4, 2003. 
[5] Akyildiz, W. Su, Y. Sankarasubramaniam and E. Cayirci, “A Survey on Sensor Networks,” IEEE Communications Magazine, Vol: 40. Issue: 8, pp. 102-114, 2002.

[6] Ji Z, "Design of an Integrated Controller Based on ZigBee Wireless Sensor Network," TELKOMNIKA Indonesian Journal of Electrical Engineering, 11(8): 4414-4421, 2013.

[7] Zhu J, Waltho A, Yang X, Guo X, "Multi-Radio Coexistence: Challenges and Opportunities," In Proceedings of the 16th International Conference on Computer Communications and Networks (ICCCN). Piscataway, NJ, USA: IEEE; 2007:358-364. 10.1109/ICCCN.2007

[8] Nga Dinh, Sangsoon Lim, “Analysis of IEEE 802.15.4 Beacon-Enabled MAC Protocol," International Journal of Electrical and Computer Engineering (IJECE), Vol. 6, No 3. June 2016.

[9] Gummadi R, Balakrishnan H, Seshan S, "Metronome: Coordinating Spectrum Sharing in Heterogeneous Wireless Networks," In Proceedings of First International Workshop on Communication Systems and Networks (COMSNETS). Piscataway, NJ, USA: IEEE; 157-166, 2009.

[10] ContikiOS, http://www.contikios.org.

[11] TelosB, http://www.memsic.com/userfiles/files/Datasheets/WSN/telosb_datasheet.pdf 